\title{
The ground state of two-hole centres in oxides
}

\author{
A M Stoneham $\dagger$, A P Pathak $+\ddagger$ and R H Bartram $\S$ \\ Theoretical Physics Division, Atomic Energy Research Establishment, Harwell, Oxfordshire \\ OXII ORA \\ $\S$ Physics Department, University of Connecticut, Storrs, Connecticut 06268, USA
}

\author{
Received 21 July 1975
}

\begin{abstract}
We discuss the nature of the ground state of the V centre in ionic oxides, where two holes are trapped by a cation vacancy. Experiment appears to indicate an $S=1$ ground state for $\mathrm{BeO}$ and $\mathrm{Al}_{2} \mathrm{O}_{3}$ and possibly $\mathrm{ZnO}$, with $S=0$ in $\mathrm{MgO}$ and $\mathrm{CaO}$. Simple theoretical arguments suggest $S=0$. We show that a weak admixture of $\left[\mathrm{O}^{\circ} \mathrm{O}^{2-}\right]$ configurations into the dominant $\left[\mathrm{O}^{-} \mathrm{O}^{-}\right]$configuration can explain the observations. Quantitative calculations are given for $\mathrm{MgO}$, and suggest the singlet state is lowest by about $30 \mathrm{~cm}^{-1}$.
\end{abstract}

\section{Introduction}

It has been well known for many years that the ground-stage of a two-electron system with an arbitrary real potential, independent of spin and velocity, is a spin singlet (Heisenberg 1928, Herring 1962, Lieb and Mattis 1962). This result allows one to understand why many two-electron colour centres (e.g. the $\mathrm{F}^{\prime}$ and $\mathrm{M}$ centres in alkali halides and the $F_{t}$ centre in alkaline earth oxides) have singlet ground states. It comes as something of a surprise to learn that two-hole centres in oxides-usually the $\mathrm{V}$ centre, where two holes are trapped next to a cation vacancy - may have a triplet ground state, since they appear to have nothing in common with known cases of ferromagnetic exchange interactions. The $\mathrm{V}$ centres have been studied in $\mathrm{MgO}$ (Wertz et al 1959, G Rius and R T Cox 1974 private communication), $\mathrm{CaO}$ (Henderson and Tomlinson 1969, Abraham et al 1975), $\mathrm{BeO}$ (Maffeo et al 1970), $\mathrm{ZnO}$ (Galland and Hervé 1970) and $\mathrm{Al}_{2} \mathrm{O}_{3}$ (Cox 1966, 1971, 1972). In all cases there is a state with spin 1 which is either the ground state or a very low-lying state. Current data indicate that the singlet $(S=0)$ is definitely lowest in $\mathrm{MgO}$ and $\mathrm{CaO}$. For $\mathrm{BeO}$ and $\mathrm{Al}_{2} \mathrm{O}_{3}$, either the triplet $(S=1)$ is lowest, or it is within $1.5 \mathrm{~cm}^{-1}$ of the ground state. The data are inconclusive for $\mathrm{ZnO}$, and are only available at $77 \mathrm{~K}$. But even here the $S=1$ state must be appreciably populated.

In the present paper we analyse the singlet-triplet splitting in $\mathrm{MgO}$. At the time these calculations were started it was believed that the $S=1$ state was lowest. Since then, the work of Rius and Cox has shown the singlet state lies lowest, a result in accord with our theory. However, it is clear from our work that systems could occur with the triplet lowest, and we describe the mechanism which makes this possible. 


\section{Basic model}

In the $\mathrm{V}$ centre in $\mathrm{MgO}$, spin-resonance work suggests a ground state in which two holes are trapped on oxygen ions on opposite sides of a cation vacancy. The centre can be described as two ions $\left[\mathrm{O}^{-} \mathrm{O}^{-}\right]$in a crystalline environment. If this description as $\mathrm{O}_{2}^{2--}$ is accurate, then a singlet state is expected. The electronic structure of a free $\mathrm{O}^{-}$ion is $1 s^{2} 2 s^{2} 2 p^{5}$. In the axial crystal field of the defect, the $p$ levels are split into one state ( $2 \sigma$ say) directed towards the cation vacancy, and two lower-energy states ( $2 \mathrm{p}_{\perp}$ say). The $2 \sigma$ orbitals on each $\mathrm{O}^{-}$contain one electron, and it is the interaction between these two electrons in the non-degenerate $\sigma$ orbitals which is of the most importance. It is then hard to think of any interaction which causes the triplet to fall below the singlet Indeed, as we show in $\S 4$, the singlet is lowest in the $\left[\mathrm{O}^{-} \mathrm{O}^{-}\right]$model. The peroxide ion, $\mathrm{O}_{2}^{2-}$, also exhibits a singlet state, although it has a much smaller $\mathrm{O}^{-}-\mathrm{O}^{-}$distance than that in the $\mathrm{V}$ centre.

The origin of a triplet state could be understood as follows. If there is weak configuration admixture from states lying at energies $\delta$ above the ground state, then the singlet and triplet energies are reduced by different amounts:

$$
E_{\mathrm{T}} \rightarrow E_{\mathrm{T} 0}-\delta_{\mathrm{T}}^{2} / \epsilon_{\mathrm{T}}, \quad E_{\mathrm{S}} \rightarrow E_{\mathrm{s} 0}-\delta_{\mathrm{S}}^{2} / \epsilon_{\mathrm{S}} .
$$

Here $E_{\mathrm{S} 0}, E_{\mathrm{T} 0}$ are the singlet and triplet energies without admixture, and $\delta_{\mathrm{S}}$ and $\delta_{\mathrm{T}}$ are the matrix elements for the admixture. Whenever $\delta_{\mathrm{T}}^{2} / \epsilon_{\mathrm{T}}$ is significantly bigger than $\delta_{\mathrm{S}}^{2} / \epsilon_{\mathrm{S}}$, the triplet will be depressed in energy by a larger amount. This is the origin of a triplet ground state. It should also be noted that, since $\left(E_{\mathrm{TO}}-E_{\mathrm{s} 0}\right)$ is very small, only very slight admixtures are needed to put the triplet lowest. These admixtures, of less than one percent, are too small to be noticed in spin resonance. We also emphasize that a triplet ground state would not violate the theorem cited in $\S 1$.

We now describe quantitative calculations for the $\mathrm{V}$ centre in $\mathrm{MgO}$. Since this work was begun it became clear that the centre did not have an $S=1$ ground state. However, it is clear that the $S=0$ and $S=1$ states lie very close in energy, and it is useful to have estimates of the various contributions to $\left(E_{\mathrm{T}}-E_{\mathrm{S}}\right)$. The calculation falls into three main parts. In the first, the wavefunctions of the oxygen ions are calculated for their different charge states and for appropriate environments. In the second, we calculate the amount by which the singlet state lies below the triplet for the $\left[\mathrm{O}^{-} \mathrm{O}^{-}\right]$configuration. Finally, we examine the effects of configuration admixture and the question whether weak admixtures of $\left[\mathrm{O}^{\circ} \mathrm{O}^{2-}\right]$ and $\left[\mathrm{O}^{2-} \mathrm{O}^{0}\right]$ are sufficient to affect the nature of the ground state.

Qualitatively, the model was first analysed by us in 1971. Quantitative calculations using Breene's (1958) $\mathrm{O}^{-}$wavefunctions were performed soon after (Stoneham, unpublished work and 1975), and gave results similar to those described here.

\section{Calculation of oxygen wavefunctions}

Three charge-states of oxygen are of in terest: $\mathrm{O}^{0}, \mathrm{O}^{-}$and $\mathrm{O}^{2-}$. We have calculated energies and wavefunctions for all three ions in several states, both singlets and triplets. Rather than calculate the structure of pairs of ions (e.g. two $\mathrm{O}^{-}$ions at the appropriate separation, or one $\mathrm{O}^{\circ}$ atom and one $\mathrm{O}^{2-}$ ion), we have recognized the large interionic distances, calculating the properties of one ion at a time, and incorporating the effects of their interaction later. This is appropriate because we seek corrections of order $10^{-4} \mathrm{eV}$ in energies which are typically several $\mathrm{eV}$. 
Table 1. Basis functions used for calculation of eigenvectors.

The exponents of the various types of atomic functions are listed; the weights of the different components were adjusted automatically by the ATMOL program. Exponents are in atomic units.

\begin{tabular}{lrrr}
\hline Type of orbital & \multicolumn{1}{c}{$0^{0}$} & \multicolumn{1}{l}{$0^{-}$} & \multicolumn{1}{c}{$0^{2-}$} \\
\hline $1 \mathrm{~s}$ & 7.6160 & 7.6126 & 7.6126 \\
& 13.3243 & 13.3654 & 13.3654 \\
$2 \mathrm{~s}$ & 1.7582 & 1.7637 & 0.5000 \\
& 2.5627 & 3.2045 & 1.7637 \\
& 4.2832 & 6.2896 & 3.2045 \\
& 5.9445 & & 6.2896 \\
$3 \mathrm{~s}$ & & & 0.6986 \\
& & & 0.8358 \\
& & & 0.9142 \\
$2 \mathrm{p}$ & 1.1536 & 0.4700 & 0.4700 \\
& 1.7960 & 0.8565 & 0.8565 \\
& 3.4379 & 1.7424 & 1.7424 \\
& 7.9070 & 3.4363 & 3.4363 \\
& & 7.8070 & 7.8070 \\
\hline
\end{tabular}

The calculations use the molecular Hartree-Fock program ATMOL (kindly provided by Dr V R Saunders of the Atlas Laboratory), and give wavefunctions for ions in a suitable point-charge environment. The point charges are essential for $\mathrm{O}^{2-}$, which is not stable as a free ion, and the changes are useful for $\mathrm{O}^{\circ}$ and $\mathrm{O}^{-}$because the asymmetry of the crystal field is included. Our choice of point-charge environment is this:

(a) In all cases the five $\mathrm{Mg}^{2+}$ nearest-neighbours are included as charges $+2|e|$ at the spacing appropriate to the perfect crystal.

(b) To obtain the correct asymmetry of the crystal field, a point charge at the other oxygen site (across the vacancy) was included. The charge was the difference between the perfect-crystal charge $-2|e|$ and the charge appropriate. Thus, for $\mathrm{O}^{-}$, another $\mathrm{O}^{-}$ ion replaces $\mathrm{O}^{2-}$, so a charge $+|e|$ is needed. For $\mathrm{O}^{2-}$, an $\mathrm{O}^{0}$ replaces $\mathrm{O}^{2-}$, and $+2|e|$ is appropriated. No extra point charge is needed for the $\mathrm{O}^{0}$ wavefunctions calculation. Thus the $\mathrm{O}^{\circ}, \mathrm{O}^{-}$and $\mathrm{O}^{2-}$ wavefunctions are calculated in an environment appropriate for the configuration in which they occur.

Reasonably extensive sets of basis orbitals were used for the wavefunctions. The

Table 2. Energy levels for different configurations.

Energy levels are in atomic units. The wavefunctions all have $\Sigma$ symmetry, and sometimes II states exist below the states given. To simplify notation, the one-electron states are written $1 \mathrm{~s}, 2 \mathrm{~s}, 2 \mathrm{p}_{\perp}, 2 \sigma, 3 \sigma$, where the $2 \sigma$ state derives mainly from the axial $2 \mathrm{p}$ state and $2 \mathrm{~s}$, etc; admixtures of $\mathrm{s}$ and $\mathrm{p}$ states are not written explicitly.

\begin{tabular}{llll}
\hline $0^{\circ}$ & ${ }^{1} \Sigma$ & $\left|\mathrm{s}^{2} 2 \mathrm{~s}^{2} 2 \mathrm{p}^{4}\right\rangle$ & -68.0545572 \\
& ${ }^{3} \Sigma$ & $\left|\mathrm{s}^{2} 2 \mathrm{~s} 2 \mathrm{p}_{\perp}^{4} 2 \sigma\right\rangle$ & -68.5292397 \\
$0^{-}$ & ${ }^{2} \Sigma$ & $\left|\mathrm{s}^{2} 2 \mathrm{~s}^{2} 2 \mathrm{p}_{\perp}^{4} 2 \sigma\right\rangle$ & -69.6919373 \\
$0^{2-}$ & ${ }^{1} \Sigma$ & $\left|\mathrm{s}^{2} 2 \mathrm{~s}^{2} 2 \mathrm{p}_{\perp}^{4} 2 \sigma^{2}\right\rangle$ & -71.0768518 \\
& ${ }^{3} \Sigma$ & $\left|\mathrm{s}^{2} 2 \mathrm{~s} 2 \mathrm{p}_{\perp}^{4} 2 \sigma^{2} 3 \sigma\right\rangle$ & -70.9799171 \\
\hline
\end{tabular}


choices, listed in table 1, were guided by those used in the calculations of Clementi (1965), Breene (1958), Watson (1958) and Harker (1974). For each ion we calculated the low-lying singlet and triplet states. The energies of these states are given in table 2. Note that we did not use Koopmans' approximation, and that excitation energies were obtained as the difference between two self-consistent calculations of total energies. No estimates of correlation are included, a point to which we return later.

\section{Calculation of $\left[0^{-} 0^{-}\right]$singlet-triplet splitting}

It has been observed by many authors, notably Herring (1962), that one of the worst ways of calculating small singlet-triplet splittings is to evaluate the two energies separately and to take the difference. Specific schemes have been proposed as alternatives. One, particularly suitable here, is that of Berezin (1972), who shows that the singlet-triplet splitting, $\Delta_{0}$ (without corrections from configuration admixture) can be written as a surface integral over the surface $x=0$ equidistant from the two oxygen ions. If $\psi_{\mathrm{L}}$ and $\psi_{\mathrm{R}}$ represent the $2 \sigma$ orbitals on the two oxygen ions, and if subscripts 1 and 2 label the electrons, then:

$$
\Delta_{0} \simeq \int \ldots \int \mathrm{d} y_{1} \mathrm{~d} z_{1} \mathrm{~d} x_{2} \mathrm{~d} y_{2} \mathrm{~d} z_{2}\left(\phi_{1} \frac{\partial \phi_{2}}{\partial x_{1}}-\phi_{2} \frac{\partial \phi_{1}}{\partial x_{1}}\right)_{x_{1}=0} .
$$

Here, if $\mathrm{s} \equiv\left\langle\psi_{\mathrm{L}} \mid \psi_{\mathrm{R}}\right\rangle$ is the overlap, then

$$
\begin{aligned}
& \phi_{1} \equiv A \psi_{\mathrm{L} 1} \psi_{\mathrm{R} 2}+B \psi_{\mathrm{L} 2} \psi_{\mathrm{R} 1} \\
& \phi_{2} \equiv B \psi_{\mathrm{L} 1} \psi_{\mathrm{R} 2}+A \psi_{\mathrm{L} 2} \psi_{\mathrm{R} 1}
\end{aligned}
$$

where $A \equiv \frac{1}{2}\left[\left(1+\mathrm{s}^{2}\right)^{-1 / 2}+\left(1-\mathrm{s}^{2}\right)^{-1 / 2}\right]$ and $B \equiv \frac{1}{2}\left[\left(1+\mathrm{s}^{2}\right)^{-1 / 2}-\left(1-\mathrm{s}^{2}\right)^{-1 / 2}\right]$. The integrand of $\Delta_{0}$ involves terms from the various basis functions in $\psi_{\mathrm{L}}$ and $\psi_{\mathrm{R}}$. For the Slater forms adopted, the individual contributions can be evaluated analytically; Berezin gives the simplest case as an example. It is straightforward to evaluate the whole of $\Delta_{0}$ by summing the contributions with correctly-weighted basis functions. We find:

$$
\Delta_{0}=1.276 \times 10^{-5} \text { au }
$$

so that the singlet lies below the triplet by about $2.8 \mathrm{~cm}^{-1}$. Even without any effects of configuration admixture, spin-resonance experiments would find it hard to discern that the true ground state was a singlet, not a triplet. Experiments on $\mathrm{MgO}$ give a splitting about an order of magnitude larger than $\Delta_{0}$.

\section{Effects of configuration admixture}

The main effect of configuration admixture is to change the singlet-triplet splitting. To discuss this quantitatively, one needs estimates of both the energy levels and the transition matrix element.

\subsection{Excited states}

The energy levels can be obtained from table 2 . There are two states of the pair of oxygens which are of main interest, and, for each of these states, both the $\left[\mathrm{O}^{\circ} \mathrm{O}^{2-}\right]$ and the 
$\left[\mathrm{O}^{2-} \mathrm{O}^{0}\right]$ configurations must be included. The states are these:

$|\mathrm{I}\rangle$, with both $\mathrm{O}^{\circ}$ and $\mathrm{O}^{2-}$ in singlet states. This state involves the transfer of an electron from the $2 \sigma$ state on one site to the $2 \sigma$ state on the other; there is no corresponding triplet excitation.

$$
\text { Excitation energy } E_{\mathrm{I}} \equiv\left[{ }^{1} E_{0}+{ }^{1} E_{2}-2^{2} E_{1}\right]=6.87 \mathrm{eV}
$$

where the energies in table 1 for an ion with charge $Q$ and spin $S$ have been written ${ }^{2 S+1} E_{-Q}$.

$|\mathrm{II}\rangle$, with $\mathrm{O}^{0}$ in a singlet state and $\mathrm{O}^{2-}$ in a triplet state. This state involves the transfer from a $2 \sigma$ orbital one one site to a $3 \sigma$ orbital on the other. The corresponding singlet excitation is not bound and has been omitted.

$$
\text { Excitation energy } E_{\mathrm{II}} \equiv\left[{ }^{1} E_{0}+{ }^{3} E_{2}-2^{2} E_{1}\right]=9 \cdot 50 \mathrm{eV} \text {. }
$$

We have also looked at other excited states in which 2 s electrons are transferred to $2 \sigma$ and $3 \sigma$ states. These excitations require over $20 \mathrm{eV}$ and have been ignored.

\subsection{Expression for the singlet-triplet splitting}

The matrix elements for the singlet and triplet admixtures differ in addition to the different excitation energies. We shall consider the matrix elements $\delta_{\mathrm{S}}$ and $\delta_{\mathrm{T}}$ in $\S 5.3$; here we want to give a basic expression for the singlet-triplet splitting and to indicate its implications.

Since we must include both $\left[\mathrm{O}^{0} \mathrm{O}^{2-}\right]$ and $\left[\mathrm{O}^{2-} \mathrm{O}^{0}\right]$ configurations, the singlet and triplet are lowered in energy by terms $-2 \delta_{\mathrm{S}}^{2} / E_{\mathrm{I}}$ and $-2 \delta_{\mathrm{T}}^{2} / E_{\mathrm{II}}$ respectively. In all, the singlet lies beneath the triplet by

$$
\Delta=\Delta_{0}+2 \delta_{\mathrm{S}}^{2} / E_{\mathrm{I}}-2 \delta_{\mathrm{T}}^{2} / E_{\mathrm{II}}
$$

It is important that $E_{\mathrm{I}}$ and $E_{\mathrm{II}}$ are comparable, for then modest differences in $\delta_{\mathrm{S}}$ and $\delta_{\mathrm{T}}$ can invert the order of the levels. In particular, the sign of $\Delta$ can easily vary from crystal to crystal.

\subsection{Matrix elements}

A separate calculation of the matrix elements is needed. There are two possible levels of detail. In the first, which we adopt, we concentrate entirely on the one-electron functions between which an electron is exchanged on going from $\left[\mathrm{O}^{-} \mathrm{O}^{-}\right]$to $\left[\mathrm{O}^{\circ} \mathrm{O}^{2-}\right]$ or $\left[\mathrm{O}^{2-} \mathrm{O}^{0}\right]$. Thus the matrix elements reduces to a sum of expressions primarily involving the $2 \sigma$ orbitals for the singlet excitations and $2 \sigma$ and $3 \sigma$ in the triplet case. A second level of detail (cf Rimmer 1964, who gives explicit expressions in another context) would include corrections because the other orbitals are slightly modified after the transfer of the electron. It would be inconsistent (as well as laborious) for us to use the fuller approach, because our calculations of $\Delta_{0}$, the splitting without configuration admixture, ignored corresponding details.

Suppose we have an approximate eigenstate $|0\rangle$, consisting of a single configuration (i.e. a single Slater determinant). The admixture of a second configuration $|1\rangle$ gives a wavefunction:

$$
|\tilde{0}\rangle=N(|0\rangle+A|1\rangle)
$$

where $A$ is given by

$$
A=-\delta / E_{10}
$$


and the change in energy is

$$
\Delta \epsilon=-\delta^{2} / E_{10}
$$

Here $N$ is a normalizing factor, and $\delta$ and $E_{10}$ are given by

$$
\begin{aligned}
& \delta=\langle 0|\mathscr{H}| 1\rangle-\langle 0 \mid 1\rangle\langle 0|\mathscr{H}| 0\rangle, \\
& E_{10}=\langle 1|\mathscr{H}| 1\rangle-\left\langle\left. 0\right|_{\mathscr{H}} \mathscr{H} \mid 0\right\rangle,
\end{aligned}
$$

where $\mathscr{H}$ is the total Hamiltonian. The values for $E_{10}$ were given earlier in this section; we are now concerned with $\delta$. Two results are useful. First, if two configurations differ by one orbital only, and if the order of the orbitals in the two determinants correspond, then (e.g. Griffith 1961)

$$
\langle 0|\mathscr{H}| 1\rangle=\left\langle k_{0}\left|\mathscr{H}_{1}\right| k_{1}\right\rangle+\sum_{i \neq \mathrm{k}}\left[\left\langle k_{0} i k_{1} i\right\rangle-\left\langle i k_{0} k_{1} i\right\rangle\right] .
$$

Here $\mathscr{H}_{1}$ is the one-electron part of the total Hamiltonian; the electron-electron interactions are included in the two-electron elements $\langle a b c d\rangle$ where the $i$ are assumed unaffected by the electron transfer from $\left|k_{0}\right\rangle$ to $\left|k_{1}\right\rangle$. The second point is that our basis orbitals have a small but finite overlap. To the desired degree, orthogonality can be ensured by the well-known modification

$$
\left|k_{0}\right\rangle \rightarrow\left|k_{0}\right\rangle-\frac{1}{2}\left\langle k_{1} \mid k_{0}\right\rangle\left|k_{1}\right\rangle
$$

and

$$
\left|k_{1}\right\rangle \rightarrow\left|k_{1}\right\rangle-\frac{1}{2}\left\langle k_{0} \mid k_{1}\right\rangle\left|k_{0}\right\rangle
$$

The contributions to the matrix element $\delta$ divide into two main parts: those involving the two oxygen ions, and those from the rest of the crystal. The dominant terms prove to be those from the rest of the crystal, outside the two oxygen ions. This part is difficult to obtain exactly, for it involves three-centre integrals which cause problems even if we represent the terms in the Hamiltonian ( $\mathscr{H}^{\prime \prime}$ say) by a sum of point-charge contributions. However, a rough estimate (actually exact for Gaussian functions) can be found in terms of the Madelung potential, corrected by omitting the contributions from the vacancy site and the two oxygen ions already treated. In the $\left\langle 0\left|\mathscr{H}^{\prime \prime}\right| 0\right\rangle$ term, the potential at an oxygen site is needed, i.e. $\left(-\alpha+\frac{1}{2}\right) Z \mathrm{e}^{2} / a$, where $\alpha$ is the Madelung constant and $Z=2$ is the ionic charge. For the $\left\langle 1\left|\mathscr{H}^{\prime \prime}\right| 0\right\rangle$ element, we assume the point-ion potential varies slowly in the region of the maximum overlap, so that it can be factored out of the matrix element. The potential at the cation vacancy is $(\alpha-2) Z \mathrm{e}^{2} / a$. In all, we have

$$
\left\langle 0\left|\mathscr{H}^{\prime \prime}\right| 1\right\rangle-\langle 0 \mid 1\rangle\left\langle 0\left|\mathscr{H}^{\prime \prime}\right| 0\right\rangle \simeq \frac{Z \mathrm{e}^{2}}{a}\langle 0 \mid 1\rangle\left(2 \alpha-\frac{5}{2}\right)
$$

where $\alpha$ is 1.7476 . The overlap $\left\langle 0^{0} 2 \sigma \mid \mathrm{O}^{2-} 2 \sigma\right\rangle$ is 0.0105 , so that this contribution to the singlet matrix element is $\delta_{\mathrm{s}}^{\prime \prime}=0.0715 \mathrm{eV}$. By contrast, the overlap $\left\langle\mathrm{O}^{0} 2 \sigma \mid \mathrm{O}^{2-3 \sigma}\right\rangle$ is much smaller, being only 0.00171 . Despite the wider spread of the $3 \sigma$ orbital, the overlap is less and the contribution to the triplet matrix element is $\delta_{\mathrm{T}}^{\prime \prime}=0.0116 \mathrm{eV}$. The part from the oxygen ions alone is straightforward, involving two-centre integrals at most. We denote this part of the Hamiltonian by $\mathscr{H}^{\prime}$. These terms have been calculated using the MIDIAT program of F R A Hopgood, based on an earlier program of Corbato and Switendick. For simplicity, the integrals in this much smaller part were calculated for a 
$\mathrm{O}^{-}-\mathrm{O}^{-}$system rather than $\mathrm{O}^{2-}-\mathrm{O}^{0}$. Thus for the admixture of $|\mathrm{I}\rangle$ we find

$$
\left\langle 0\left|\mathscr{H}^{\prime}\right| 1\right\rangle=0.0098 \mathrm{eV}
$$

and

$$
-\langle 0 \mid 1\rangle\left\langle 0\left|\mathscr{H}^{\prime}\right| 0\right\rangle=0.0280 \mathrm{eV} .
$$

The total matrix element for the singlet admixture is the sum of contributions (5.11) and $(5.12), \delta_{\mathrm{s}}=0.109 \mathrm{eV}$. This admixture leads to a lowering of the singlet state by

$$
2 \delta_{\mathrm{s}}^{2} / E_{1}=27.9 \mathrm{~cm}^{-1} \text {. }
$$

By contrast, the triplet is negligibly lowered $\left(2 \delta_{\mathrm{T}}^{2} / E_{\mathrm{II}}<1 \mathrm{~cm}^{-1}\right)$. This is partly a result of the small overlap and partly because $\delta_{\mathrm{T}}^{\prime}$ and $\delta_{\mathrm{T}}^{\prime \prime}$ prove to have opposite signs, giving some cancellation. However, the smallness of $\delta_{\mathrm{T}}$ is clearly a special feature of $\mathrm{MgO}$, and the detailed results suggest that in other systems $\delta_{\mathrm{S}}$ and $\delta_{\mathrm{T}}$ could easily be comparable.

\subsection{Comparison with experiment}

Collecting together the results of (4.4), (5.3) and (5.13), the singlet state should be lowest in $\mathrm{MgO}$ by

$$
\Delta=30 \cdot 7 \mathrm{~cm}^{-1}
$$

a singlet-triplet splitting which is dominated by configuration-admixture of higher singlet states.

The splitting has not yet been measured accurately, but R T Cox and G Rius (private communication) have shown that the triplet state lies above the singlet in $\mathrm{MgO}$, and that the population of the triplet state becomes negligible as one lowers the sample temperature from $20 \mathrm{~K}$ to $6 \mathrm{~K}$. Both these results agree with our prediction (5.14).

A second point to check concerns $D$, the zero-field splitting in the triplet state. Experiment gives a value very close to that expected from dipole-dipole interactions within $\left[\mathrm{O}^{-} \mathrm{O}^{-}\right]$. If there are admixtures of $\left[\mathrm{O}^{0} \mathrm{O}^{2-}\right]$, then some changes will result. We find that the admixture leads to a negligible change in $D$. The change has the form $\left(\delta_{\mathrm{T}} / E_{\mathrm{II}}\right)^{2} \rho$, where $\delta_{\mathrm{T}} / E_{\mathrm{II}}$ is a measure of the admixture and $\rho$ is the spin-spin interaction within the triplet $\mathrm{O}^{2-}$. Since $\delta_{\mathrm{T}}$ is very small $\left(\delta_{\mathrm{T}} / E_{\mathrm{II}}<10^{-4}\right)$ and $\rho$ (whilst not accurately known) will be a few $\mathrm{cm}^{-1}$ at most, the change in $D$ is less than $10^{-7} \mathrm{~cm}^{-1}$. This should be compared with the experimental value of $0.021 \mathrm{~cm}^{-1}$ (Henderson and Wertz 1968).

\section{Conclusions}

We have examined the effects of a weak configuration admixture of $\left[\mathrm{O}^{0} \mathrm{O}^{2-}\right]$ and $\left[\mathrm{O}^{2-} \mathrm{O}^{0}\right]$ into the dominant $\left[\mathrm{O}^{-} \mathrm{O}^{-}\right]$configuration in $\mathrm{MgO}$. Our conclusion is that the configuration admixture dominates in the singlet-triplet splitting, giving a value consistent with experiment both in sign and magnitude. The degree of admixture is also consistent with the spin-resonance data for the triplet.

The results for $\mathrm{MgO}$ have an important implication for other crystals, for $\delta_{\mathrm{T}}$ is small because of special cancellations associated with the precise form of the $3 \sigma$ wavefunction for $\mathrm{O}^{2-}$. In other cases, $\delta_{\mathrm{S}}$ and $\delta_{\mathrm{T}}$ could easily be comparable. Since the excitation energies $E_{\mathrm{I}}$ and $E_{\mathrm{fI}}$ are comparable, it is perfectly possible that the triplet should be the 
ground state in some crystals. However, the singlet-triplet splitting should be small in any case, allowing an appreciable population of the triplet state, even when the singlet lies lowest.

\section{Acknowledgments}

It is a pleasure to acknowledge stimulating discussions with $\operatorname{Dr} \mathrm{R} T \mathrm{Cox}$, who drew our attention to this problem, and the hospitality to two of us (A M S and R H B) of Dr P Servoz-Gavin and his group at the Centre d'Etudes Nucleaires in Grenoble where some of the original ideas were formulated. Further discussions with Dr A H Harker were valuable in clarifying a number of aspects. We are also greatly indebted to DrV R Saunders for making available the ATMOL program.

\section{References}

Abraham M M, Chen Y, Boatner L A and Reynolds R W 1975 Solid St. Commun. 16 1209-13

Berezin A 1972 Phys. Stat. Solidi (b) 4951

Breene R G 1958 Phys. Rev. 1111111

Clementi E 1965 IBM J. Res. Dev. suppl 9

Cox R T 1966 Proc. XIV Colloque Ampere, Ljubljana ed R Blinc (Amsterdam: North-Holland) 279-83

- 1971 Solid St. Commun. 91989

1972 Thesis University of Grenoble pp 306-7

Galland D and Hervé A 1970 Phys. Lett. 33A 1-2

Griffith J S 1961 Theory of Transition Metal Ions (Cambridge: Cambridge UP)

Harker A H 1974 AERE Report TP568

Heisenberg W 1928 Z. Phys. 49619

Henderson B and Tomlinson A C 1969 J. Phys. Chem. Solids 301801

Henderson B and Wertz J E 1968 Adv. Phys. 17 749-855

Herring C 1962 Rev. Mod. Phys. 34631

Lieb E and Mattis D 1962 Phys. Rev. 125104

Maffeo B, Hervé A and Cox R T 1970 Solidi St. Commun. $82169-72$

Rimmer D E 1964 Proc. Int. Conf. on Magnetism (London and Bristol: Institute of Physics) pp 337-41

Stoneham A M 1975 Theory of Defects in Solids $\S 16.4$ (Oxford: Oxford UP)

Watson R E 1958 Phys. Rev. 1111108

Wertz J E, Auzins P, Griffiths J H E and Orton J W 1959 Discuss. Faraday Soc. 28136 